\title{
KINEMATIC PROPERTIES OF DOUBLE-BARRED GALAXIES: SIMULATIONS VERSUS INTEGRAL-FIELD OBSERVATIONS
}

\author{
Min Du ${ }^{1}$, Victor P. Debattista ${ }^{2}$, Juntai Shen ${ }^{1}$, and Michele Cappellari ${ }^{3}$ \\ ${ }^{1}$ Key Laboratory of Research in Galaxies and Cosmology, Shanghai Astronomical Observatory, Chinese Academy of Sciences, 80 Nandan Road, Shanghai 200030, \\ China; jshen@shao.ac.cn, vpdebattista@gmail.com \\ 2 Jeremiah Horrocks Institute, University of Central Lancashire, Preston, PR1 2HE, UK \\ ${ }^{3}$ Sub-department of Astrophysics, Department of Physics, University of Oxford, Denys Wilkinson Building, Keble Road, Oxford OX1 3RH, UK \\ Received 2016 January 29; revised 2016 June 9; accepted 2016 June 24; published 2016 August 23
}

\begin{abstract}
Using high-resolution $N$-body simulations, we recently reported that a dynamically cool inner disk embedded in a hotter outer disk can naturally generate a steady double-barred (S2B) structure. Here we study the kinematics of these S2B simulations, and compare them to integral-field observations from ATLAS ${ }^{3 D}$ and SAURON. We show that S2B galaxies exhibit several distinct kinematic features, namely: (1) significantly distorted isovelocity contours at the transition region between the two bars, (2) peaks in $\sigma_{\mathrm{LOS}}$ along the minor axis of inner bars, which we term " $\sigma$-humps," that are often accompanied by ring/spiral-like features of increased $\sigma_{\text {LOS }}$, (3) $h_{3}-\bar{v}$ anticorrelations in the region of the inner bar for certain orientations, and (4) rings of positive $h_{4}$ when viewed at low inclinations. The most impressive of these features are the $\sigma$-humps; these evolve with the inner bar, oscillating in strength just as the inner bar does as it rotates relative to the outer bar. We show that, in cylindrical coordinates, the inner bar has similar streaming motions and velocity dispersion properties as normal large-scale bars, except for $\sigma_{z}$, which exhibits peaks on the minor axis, i.e., humps. These $\sigma_{z}$ humps are responsible for producing the $\sigma$-humps. For three well-resolved early-type S2Bs (NGC 2859, NGC 2950, and NGC 3941) and a potential S2B candidate (NGC 3384), the S2B model qualitatively matches the integral-field data well, including the " $\sigma$-hollows" previously identified. We also discuss the kinematic effect of a nuclear disk in S2Bs.
\end{abstract}

Key words: galaxies: individual (NGC 2859, NGC 2950, NGC 3941, NGC 3384) - galaxies: kinematics and dynamics - galaxies: structure

\section{INTRODUCTION}

Optical and infrared observations have shown that approximately one-third of early-type barred galaxies host a misaligned inner bar (also termed "secondary bar"; Erwin \& Sparke 2002; Laine et al. 2002; Erwin 2004). Steady doublebarred (S2B) structures are also seen in later Hubble types, though we still lack systematic statistics because of the stronger dust extinction in their central regions (Erwin 2005). Numerical simulations are powerful tools for studying the formation and evolution of such multi-bar structures. Previous $N$-body +hydrodynamical simulations suggested that gas dissipation plays a vital role in inducing and maintaining an inner bar (e.g., Friedli \& Martinet 1993; Shlosman \& Heller 2002; Englmaier \& Shlosman 2004). However, the observation of galaxies without a large amount of gas (Petitpas \& Wilson 2004) indicated that gas might not be the key ingredient to maintaining, or even forming, S2Bs. Increasingly, $N$-body simulations have successfully formed S2Bs without the requirement of gas (Rautiainen \& Salo 1999; Rautiainen et al. 2002; Debattista \& Shen 2007; Heller et al. 2007; Saha \& Maciejewski 2013; Du et al. 2015). Nevertheless, the essential initial conditions by which S2Bs form is still unclear. In Du et al. (2015), we explored a large parameter space of the mass, dynamical temperature (Toomre- $Q$ ), and thickness of the stellar disk in isolated pure-disk three-dimensional (3D) $N$-body simulations. Our simulations suggested that a dynamically cool inner disk can naturally trigger small-scale bar instabilities leading to S2Bs, without the need for gas. This result is also consistent with the result of Wozniak (2015), who successfully formed long-lived S2Bs with $N$-body+hydrodynamical simulations in which a nuclear disk forming from accumulated gas followed by star formation, which plays an important role in generating the inner bar. This scenario is also consistent with the recent observation of NGC 6949 that the size of the starburst nuclear molecular disk matches well with the size of the inner bar (Romeo \& Fathi 2015).

Observations (Buta \& Crocker 1993; Friedli \& Martinet 1993; Corsini et al. 2003) suggest that the two bars in an S2B rotate independently, which is also found in numerical simulations (e.g., Debattista \& Shen 2007; Shen \& Debattista 2009; Saha \& Maciejewski 2013; Du et al. 2015; Wozniak 2015). Instead of being rigid bodies, the amplitudes and pattern speeds oscillate as the two bars rotate through each other (Debattista \& Shen 2007; Du et al. 2015), which is consistent with the loop-orbit predictions of Maciejewski \& Athanassoula (2007; see also Maciejewski \& Sparke 1997, 2000; Maciejewski \& Athanassoula 2008; Maciejewski \& Small 2010). Such dynamically decoupled inner bars in S2Bs have been hypothesized to be a mechanism for driving gas past the inner Lindblad resonance of outer bars to feed supermassive black holes that power active galactic nuclei (Shlosman et al. 1989, 1990).

Two-dimensional integral-field unit (IFU) spectroscopy provides a very powerful method for studying bars from a kinematic point of view. Several kinematic signatures of bars have been predicted and observed. Many theoretical analyses (e.g., Miller \& Smith 1979; Vauterin \& Dejonghe 1997; Bureau \& Athanassoula 2005) have shown that bars twist the mean velocity $(\bar{v})$ fields because of significant radial streaming motions, thus making the kinematic major axis misaligned with the photometric major axis of the whole disk. For both stars and gas, the kinematic major axis generally turns toward the 
opposite direction with respect to the major axis of bars. IFU observations of the early-type galaxies have shown that barred galaxies are more likely to generate larger kinematic misalignments than unbarred galaxies (Cappellari et al. 2007; Krajnović et al. 2011). The central elliptical velocity dispersion $(\sigma)$ peak should be aligned with the orientation of the large-scale bar (Miller \& Smith 1979; Vauterin \& Dejonghe 1997). Over the extent of the bar, the third order Gauss-Hermite moment $\left(h_{3}\right)$ is correlated with $\bar{v}$ in edge-on views (Bureau \& Athanassoula 2005). In face-on views, a minimum in $h_{4}$ is present when a boxy/peanut (B/P) bulge exists (Debattista et al. 2005; Méndez-Abreu et al. 2014).

We know little about the kinematic properties of S2Bs. The misalignment between the kinematic major axis and the photometric major axis has also been expected to be observed in $\bar{v}$ fields of S2Bs (Chevalier \& Furenlid 1978; Moiseev \& Mustsevoi 2000). However, Moiseev et al. (2004) found the twists due to the inner bar on the stellar velocity field are quite small compared with the twists in gaseous kinematics, which led them to question the existence of decoupled inner bars. Shen \& Debattista (2009) showed that twists due to inner bars are smaller than previously expected, thus the kinematics of S2Bs can still be consistent with observations of Moiseev et al. (2004). de Lorenzo-Cáceres et al. (2008) studied 2D stellar velocity and velocity dispersion maps of four S2Bs (NGC 2859, NGC 3941, NGC 4725, and NGC 5850) with the SAURON IFU. Surprisingly, the velocity dispersion maps revealed two local minima, which they termed " $\sigma$-hollows," located near the ends of the inner bar in each galaxy (see also de Lorenzo-Cáceres et al. 2012). They proposed that $\sigma$-hollows occur as a result of the contrast between the velocity dispersion of a hotter bulge and the inner bar, which is dominated by ordered motions and thus has a low $\sigma$. The S2B model of Shen \& Debattista (2009) also exhibited a misalignment between the inner bar and the velocity dispersion.

Self-consistent numerical models are very powerful tools for understanding the dynamics and kinematics of S2Bs. In Du et al. (2015), we were able to form S2Bs from pure disks; we summarize these results in Section 2.1. In this paper, we analyze the kinematics of the S2B model. We introduce the Voronoi binning method used in extracting the kinematics in Section 2.2. In Section 3, we show that the S2B model qualitatively matches well with the kinematics of S2Bs in the ATLAS $^{3 \mathrm{D}}$ (Cappellari et al. 2011) and SAURON (Emsellem et al. 2004) surveys, especially for the $\sigma$-humps/hollows. The detailed kinematic analyses of the S2B model are presented in Section 4. In Section 5, we discuss the kinematic effects of a nuclear disk in the S2Bs. Finally, our conclusions are summarized in Section 6.

\section{METHOD}

\subsection{Models}

A detailed description of the initial conditions and evolution of our self-consistent $\mathrm{N}$-body models has been presented in $\mathrm{Du}$ et al. (2015). Here we give a brief introduction to the models we evolved with a 3D cylindrical polar grid code, GALAXY (Sellwood \& Valluri 1997; Sellwood 2014). The S2B model studied here is the standard S2B model from Du et al. (2015). It starts from an isolated exponential disk that is located at the center of a rigid logarithmic halo. The initial disk has $4 \times 10^{6}$ equal-mass particles, softened with a 0.01 length unit (the unit of length is the initial disk scale-length). In the outer regions of the disk, the dynamical temperature parameter (Toomre- $Q$ ) is roughly constant at 2.0 , while it is gradually reduced to 0.5 at the center, i.e., the central value of Toomre- $Q b_{Q}=0.5$. The dynamically cool inner disk generates a bar instability separate from the one in the outer part, resulting in a double-barred structure. As shown in Figure 2 in Du et al. (2015), the morphology of the newly formed inner bar is quite rectangular, or even peanut-like. After the S2B structure forms, the amplitude and morphology of the inner bar continue evolving, due to the interaction between the two bars, until they reach a roughly steady state. During the steady state phase, the amplitude of the inner bar is relatively steady, and its morphology becomes oval-like. The S2B structure rotates steadily in a now hotter disk, similar to a lenticular (S0) galaxy. We analyze the kinematic properties of the S2B model in the steady state phase.

The scaling to physical units is obtained by setting the mass unit to $M_{0}=8.0 \times 10^{10} M_{\odot}$ and the length unit to $R_{d}=$ $2.5 \mathrm{kpc}$, which gives a time unit of $T_{0} \simeq 6.6 \mathrm{Myr}$ and a velocity unit of $371 \mathrm{~km} \mathrm{~s}^{-1}$. For the S2B model, the total mass of the disk is $M_{d}=1.5 M_{0}=1.2 \times 10^{11} M_{\odot}$, extending to about $\sim 15 \mathrm{kpc}$. The rigid potential of the halo provides a flat rotation curve at $V_{c} \sim 0.6$, corresponding to $222 \mathrm{~km} \mathrm{~s}^{-1}$. All analyses are made at $T>290 \simeq 1.9 \mathrm{Gyr}$ when the two bars have reached a steady state, during which the kinematics do not evolve much. The rotation periods of the two bars are stable at $P_{\text {inner }} \sim 12.8(\sim 84.5 \mathrm{Myr})$ and $P_{\text {outer }} \sim 35.1(\sim 231.7 \mathrm{Myr})$. Thus the inner bar rotates roughly three times faster than its outer counterpart, $P_{\text {inner }} / \mathrm{P}_{\text {outer }} \sim 0.36$. Measured by tracing half-way down the peak of bar amplitudes, the semimajor axes of the outer bar and the inner bar are $a_{\text {outer }} \sim 3.0(\sim 7.5 \mathrm{kpc})$ and $a_{\text {inner }} \sim 0.3(\sim 0.75 \mathrm{kpc})$, respectively. For comparison purposes, we also present a single-barred (SB) model. Using $b_{Q}=0.8$, the initial nuclear regions of the SB model are not as cool as in the S2B model, so the inner disk only triggers one bar instability leading to a single bar. The bar in the SB model has a similar semi-major axis $\left(a_{B} \sim 7.5 \mathrm{kpc}\right)$ and pattern speed $\left(P_{B} \sim 223.1 \mathrm{Myr}\right)$ as the outer bar in the S2B model.

\subsection{Extracting Kinematics}

A unique advantage of simulations is that we can project the simulated galaxy to any desired orientation. To extract reliable kinematics, including the high-order Gauss-Hermite moments $h_{3}$ and $h_{4}$ (Gerhard 1993; van der Marel \& Franx 1993), the signal-to-noise ratio $(\mathrm{S} / \mathrm{N}) \geqslant 50$ is usually considered necessary. For simulations, given that the number of particles follows Poissonian statistics, the required $\mathrm{S} / \mathrm{N}$ can be transformed into a requirement on the number of particles $\left(N_{p}\right)$ in each bin $\left(\mathrm{S} / \mathrm{N}=\sqrt{N_{p}}\right)$. Here we apply the widely used Voronoi binning method (Cappellari \& Copin 2003) to bin particles in such a way that each bin typically contains at least 2500 particles. Then we bin the particles in velocity space, with 50 velocity bins, and fit the resulting synthetic line-of-sight velocity distribution (LOSVD) with a Gauss-Hermite parametrization $\left(\bar{v}, \sigma, h_{3}\right.$, and $\left.h_{4}\right)$. We have checked that $\mathrm{S} / \mathrm{Ns}$ larger than 50 give consistent measurements of $h_{3}$ and $h_{4}$. 


\section{A GENERAL COMPARISON OF KINEMATIC PROPERTIES WITH THE DOUBLE-BARRED GALAXIES IN THE ATLAS ${ }^{3 D}$ AND SAURON SURVEYS}

The S2B model is quite similar to massive early-type galaxies, so it can be compared with S2Bs observed in the ATLAS $^{3 D}$ and SAURON surveys. According to the S2B catalog of Erwin (2004) and Erwin \& Sparke (2003), seven early-type S2Bs were observed in the ATLAS ${ }^{3 D}$ survey, namely NGC 2859, NGC 2950, NGC 3941, NGC 2962, NGC 4340, NGC 3945, and NGC 7280. The kinematics of three S2Bs are shown in Figure 1. From top to bottom, they are NGC 2859, NGC 3941, and NGC 2950, followed by the best matching model time step. Their inner bars are well confirmed by ellipse fitting and unsharp mask on their infrared images. Based on the observed ellipticity of the outer disks, their inclination angles are $i \sim 33^{\circ}, 58^{\circ}$, and $62^{\circ}$, respectively, which are obtained from HyperLeda. NGC 3384 is not a well confirmed S2B candidate. Fisher \& Drory (2010) identified the inner bar on the infrared image by a photometric decomposition of the bulge region. In previous studies (Erwin \& Sparke 2003; Erwin 2004; Sarzi et al. 2006), NGC 3384 was classified as a single-barred galaxy, with the inner component identified as a nuclear disk by an unsharp mask. In this paper, we consider NGC 3384 as a potential S2B candidate, and present some kinematic evidence for the existence of the inner bar (Figure 2). The inclination angle is $\sim 61^{\circ}$ (Erwin 2004).

The S2B model is inclined to the same inclination as each target $\mathrm{S} 2 \mathrm{~B}$ at the time that the two bars have the same relative orientation during the steady state. The short black, long black, and red dashed lines refer to the orientations of the inner bar, outer bar, and the line-of-nodes (LON) of the disk, respectively, as given in Erwin \& Sparke (2003) and Erwin (2004). The central kinematics $(R \leqslant 20$ arcsec) are well resolved in all these galaxies. The logarithmically spaced isophotes of the S2B galaxies in Figure 1 are plotted using the $R$-band observations of Erwin \& Sparke (2003). As shown in Figures 1 and 2, the S2B model qualitatively matches well with the kinematics of the observations, especially for $\sigma$ and $h_{4}$. It is worth emphasizing that only one S2B model, at different epochs, is used to model the observations of different galaxies. We do not expect to match these galaxies in every aspect with such a simple model. Rather, we are interested in the qualitative similarities between the model and observed galaxies.

As shown in Figures 1 and 2, the most impressive kinematic feature is the $\sigma$ enhancements, which we term " $\sigma$-humps," appearing along the minor axis of the inner bar. For NGC 2859 and NGC 2950, $\sigma$-humps are accompanied by moderate $\sigma$ ring/ spiral-like features. NGC 3384 also exhibits $\sigma$-humps, consistent with the existence of an inner bar that is parallel to the LON. Relatively lower $\sigma$ at the ends of the inner bar can partially explain the $\sigma$-hollows found by de Lorenzo-Cáceres et al. (2008). We have confirmed that the initial disk does not exhibit such $\sigma$-humps/hollows, and the $\sigma$-humps develop with the $\mathrm{S} 2 \mathrm{~B}$ formation.

The qualitative agreement of high-order Gauss-Hermite moments is particularly impressive for NGC 2859 and NGC 2950 , especially the positive $h_{4}$ rings appearing over the projected regions of the inner bar. These galaxies are the ones that have a velocity dispersion that is better resolved by the ATLAS ${ }^{3 D}$ data. As noted in Cappellari et al. (2011), for typical velocity dispersions $\sigma \lesssim 120 \mathrm{~km} \mathrm{~s}^{-1}$, the LOSVD is not well resolved and the Gauss-Hermite moments are gradually penalized by $\operatorname{PPXF}($ Cappellari \& Emsellem 2004) to suppress the noise in the extracted kinematics. This likely explains the less clear structure in the $h_{3}$ and $h_{4}$ maps of NGC 3941. Furthermore, NGC 2859 and NGC 2950 present clear signatures of fast-rotating nuclear disks, i.e., significant local maxima and minima in the $\bar{v}$ fields and $h_{3}-\bar{v}$ anti-correlations (see also Section 4.3) along the LON close to the center. This result is consistent with the analysis of NGC 2859 presented in Erwin et al. (2015) and de Lorenzo-Cáceres et al. (2013). To better show the relative importance of rotation and velocity dispersion, we plot the deprojected $|v| / \sigma$ in Figure 3. A rotation-dominated nuclear disk generates $\left|v_{\mathrm{dp}}\right| / \sigma>1$ along the kinematic major axis, where $v_{\mathrm{dp}}=\bar{v} / \sin i$. It is clear that both NGC 2859 and NGC 2950 also have a rotation-dominated nuclear disk within 10 arcsec, in addition to the inner bar. As shown in the bottom panels of Figure 3, without any dynamically cold nuclear disk, the central region of the S2B model is significantly dominated by velocity dispersion, $\left|v_{\text {dp }}\right| / \sigma \sim 0.7$. Thus, in NGC 2859 and NGC 2950 the central features of $\bar{v}$ and $h_{3}$ are more likely to be dominated by the nuclear disk. It is reasonable that the S2B model does not match the $\bar{v}$ and $h_{3}$ fields perfectly. NGC 3384 shows moderate rotation in the central region, $\left|v_{\mathrm{dp}}\right| / \sigma \sim 0.8$, thus it is unclear whether a nuclear disk exists or not.

In conclusion, the S2B model is able to qualitatively match many of the kinematics of observed S2Bs, making it very useful for studying the kinematics of S2Bs. In the following section, we use this model to analyze these kinematic properties in detail.

\section{KINEMATIC ANALYSES}

\section{1. $\bar{v}$ Twists}

In Figure 4, we show $\bar{v}$ fields for the S2B and SB models. Aligned with the LON (here the $x$-axis), the large-scale bar in the SB model shows smooth and nearly parallel isovelocity contours in the region of the bar (the leftmost panel, inclination $i=45^{\circ}$ ), as expected. The other three panels show $\bar{v}$ fields of the $\mathrm{S} 2 \mathrm{~B}$ model when the relative position angle of the two bars $\left(\mathrm{PA}_{\text {rel }}=\left|\mathrm{PA}_{\text {inner }}-\mathrm{PA}_{\text {outer }}\right|\right)$ is $\sim 0^{\circ}, 45^{\circ}$, and $90^{\circ}$, respectively. For the S2B model, $\bar{v}$ fields are similar to those of the $\mathrm{SB}$ model in the large-scale bar regions because the large-scale bars in the S2B and SB models rotate at nearly the same pattern speed. At the very central regions where the inner bar dominates, the isovelocity contours even break up, forming local minima and maxima, especially the inner bar is perpendicular to the $\mathrm{LON}$ (the rightmost panel, $\mathrm{PA}_{\text {rel }}=90^{\circ}$, $i=45^{\circ}$ ). In the case of an inclined axisymmetric nuclear disk, the kinematic axis must align with the LON. Since stars in bar regions have significant radial streaming motions, the kinematic axis is expected to be misaligned from the LON. As shown in Figure 4, when $\mathrm{PA}_{\text {rel }}=45^{\circ}$, within the projected regions of the inner bar, the isovelocity contours are slightly distorted toward the opposite direction of the major axis of the inner bar, in agreement with Shen \& Debattista (2009). Thus it might be because the twists are too weak that the kinematic axis of the observed galaxies (Figures 1 and 2) does not exhibit a clear misalignment with the LON in the central regions. Furthermore, as presented in Section 3, NGC 2859 and NGC 2950 have a rotation-dominated nuclear disk which also significantly weakens the misalignment. Therefore, it is not surprising that previous observations (Moiseev et al. 2004) did 

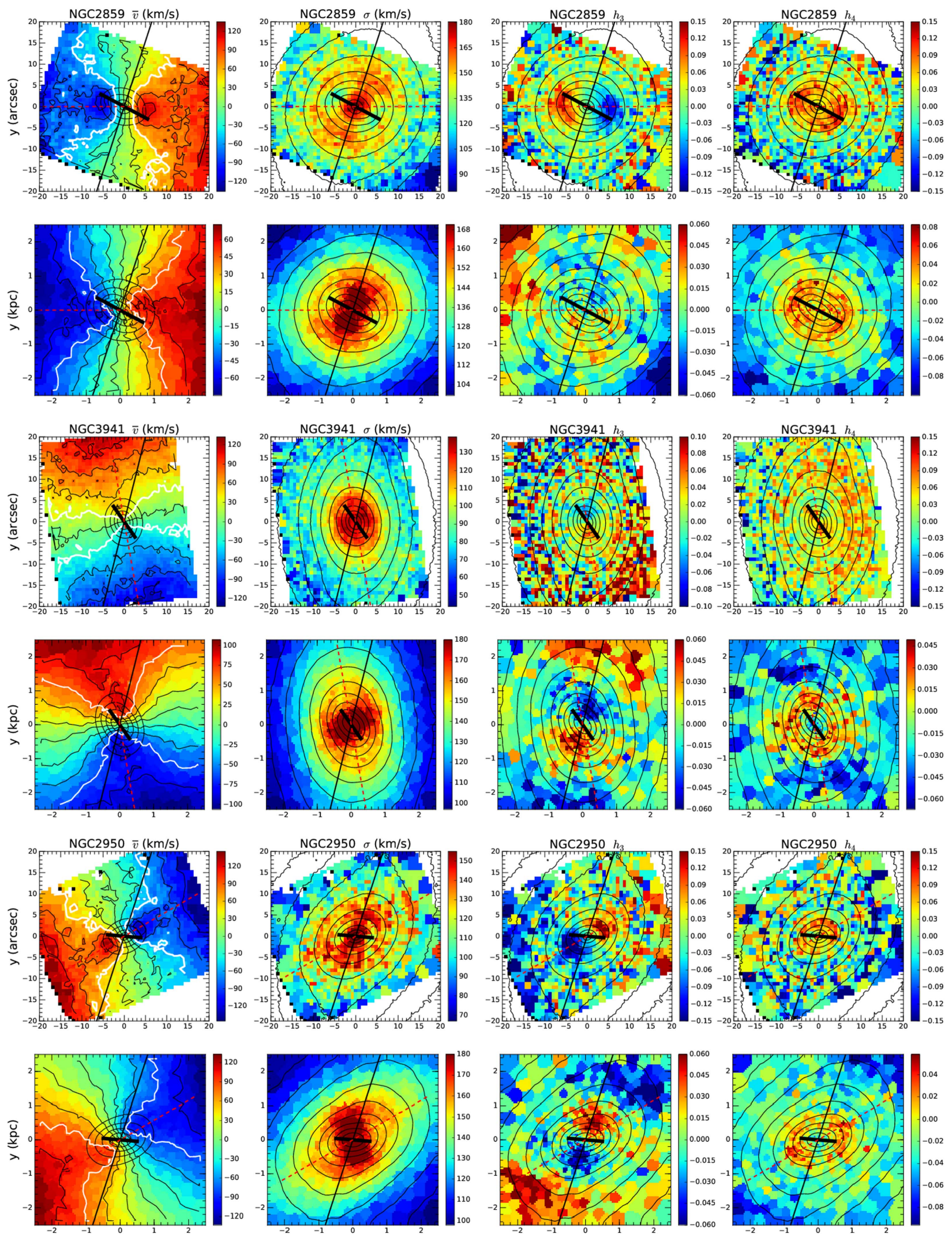

Figure 1. Kinematic maps of three S2B galaxies (NGC 2859, 3941, and 2950) in the ATLAS ${ }^{3 D}$ survey (Cappellari et al. 2011) followed by the best matching S2B model with similar orientations for the two bars and an identical inclination angle. In all panels, the model is cropped to the same area $x, y \in[-2.5,2.5] \mathrm{kpc}$, in which case the size of the inner bars are similar in the panels presenting the model and the observations. The Gauss-Hermite moments of the LOSVDs are shown from left to right: $\bar{v}, \sigma, h_{3}$, and $h_{4}$. Logarithmic isodensity contours are overlaid in black. For the $\bar{v}$ maps, the isovelocity contours and the central isodensity contours are overlaid to show the twists of isovelocity contours caused by inner bars. A few significantly distorted isovelocity contours are highlighted with white curves. Red dashed lines indicate the orientations of the LON. The short black and long black lines roughly show the lengths and orientations of the inner and outer bars, respectively. 

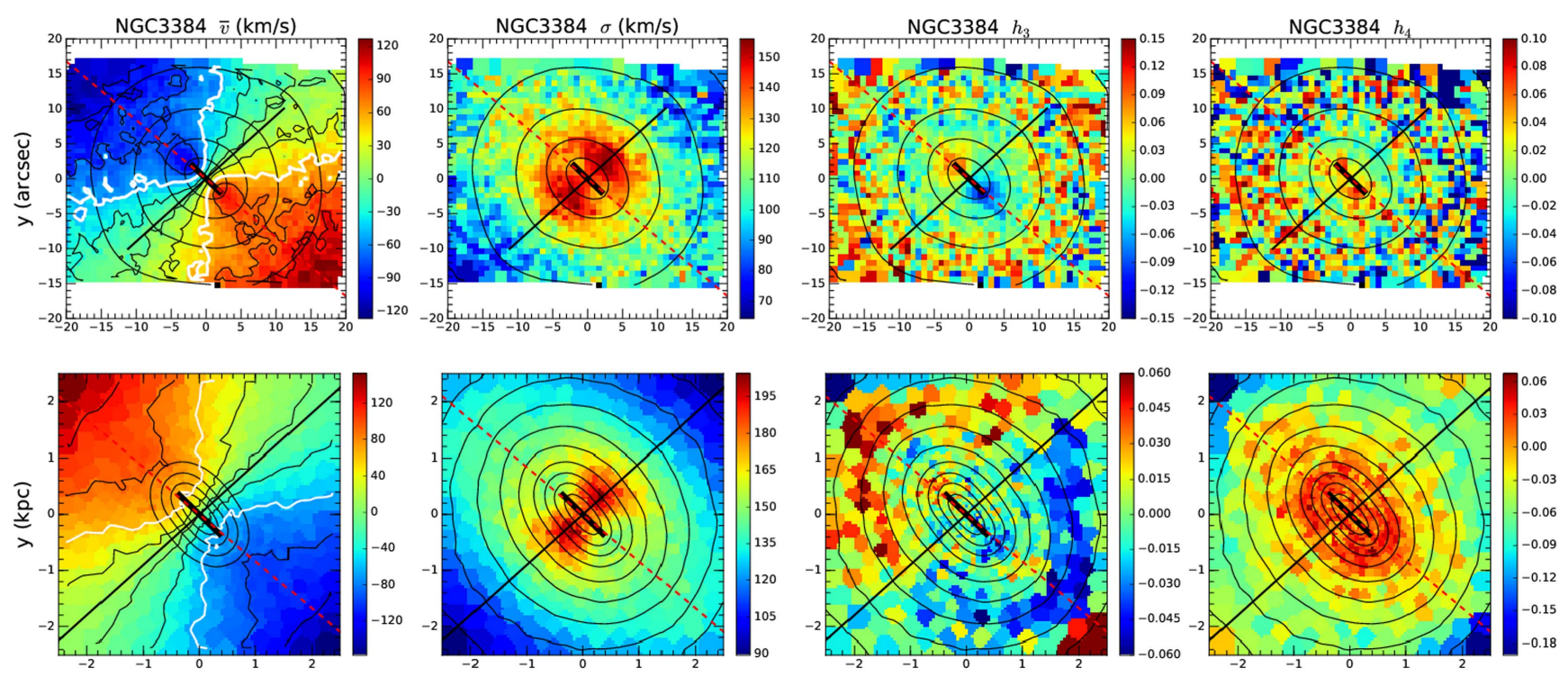

Figure 2. Kinematic maps of NGC 3384, a potential S2B candidate obtained from the SAURON survey (Emsellem et al. 2004) followed by the best matching S2B model.
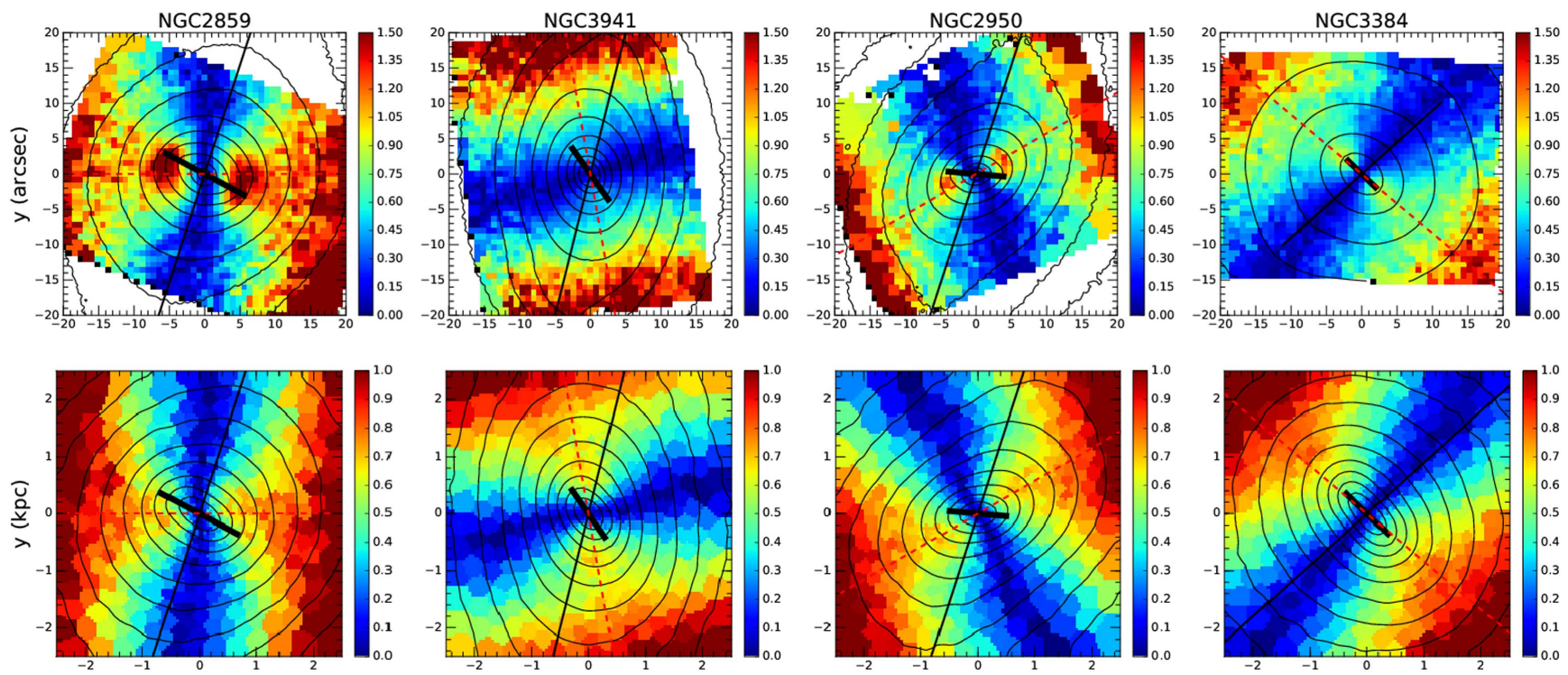

Figure 3. Maps of $\left|v_{\mathrm{dp}}\right| / \sigma$. From left to right: NGC 2859, 3941, 2950, and 3384, followed by the best matching S2B model.

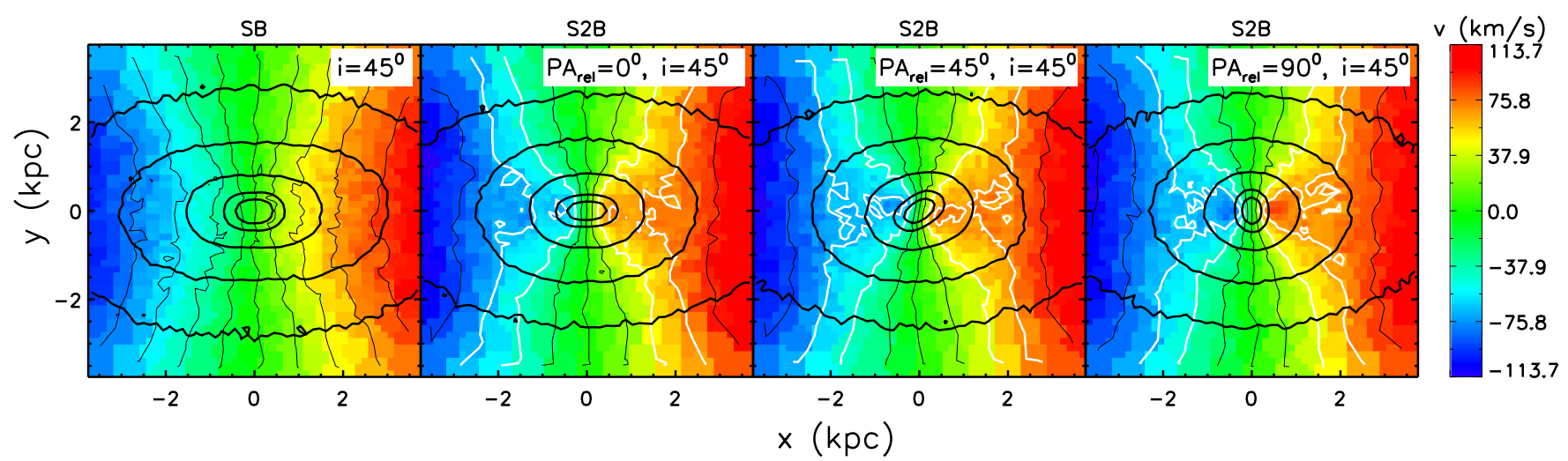

Figure 4. $\bar{v}$ maps of the SB model (the leftmost panel) and the S2B model, showing the twists caused by a rapidly rotating inner bar. Highlighted with white contours, the $\bar{v}$ contours show that the most significant twists are present at an intermediate region between the two bars. The outer bar is fixed along the LON ( $x$-axis). For the S2B model, the relative angle $\mathrm{PA}_{\text {rel }}$ between the inner bar and the outer bar is $0^{\circ}, 45^{\circ}$, and $90^{\circ}$, from left to right. The disk is inclined at $i=45^{\circ}$. The logarithmic isodensity contours (levels $0.9,0.8,0.6,0.4,0.2$ of $\ln \left(\Sigma_{\max } / \Sigma_{\min }\right)$ ) are overlaid in black. The contours at levels 0.2 and 0.8 roughly coincide with the photometric edge of the outer bar and the inner bar, respectively. 


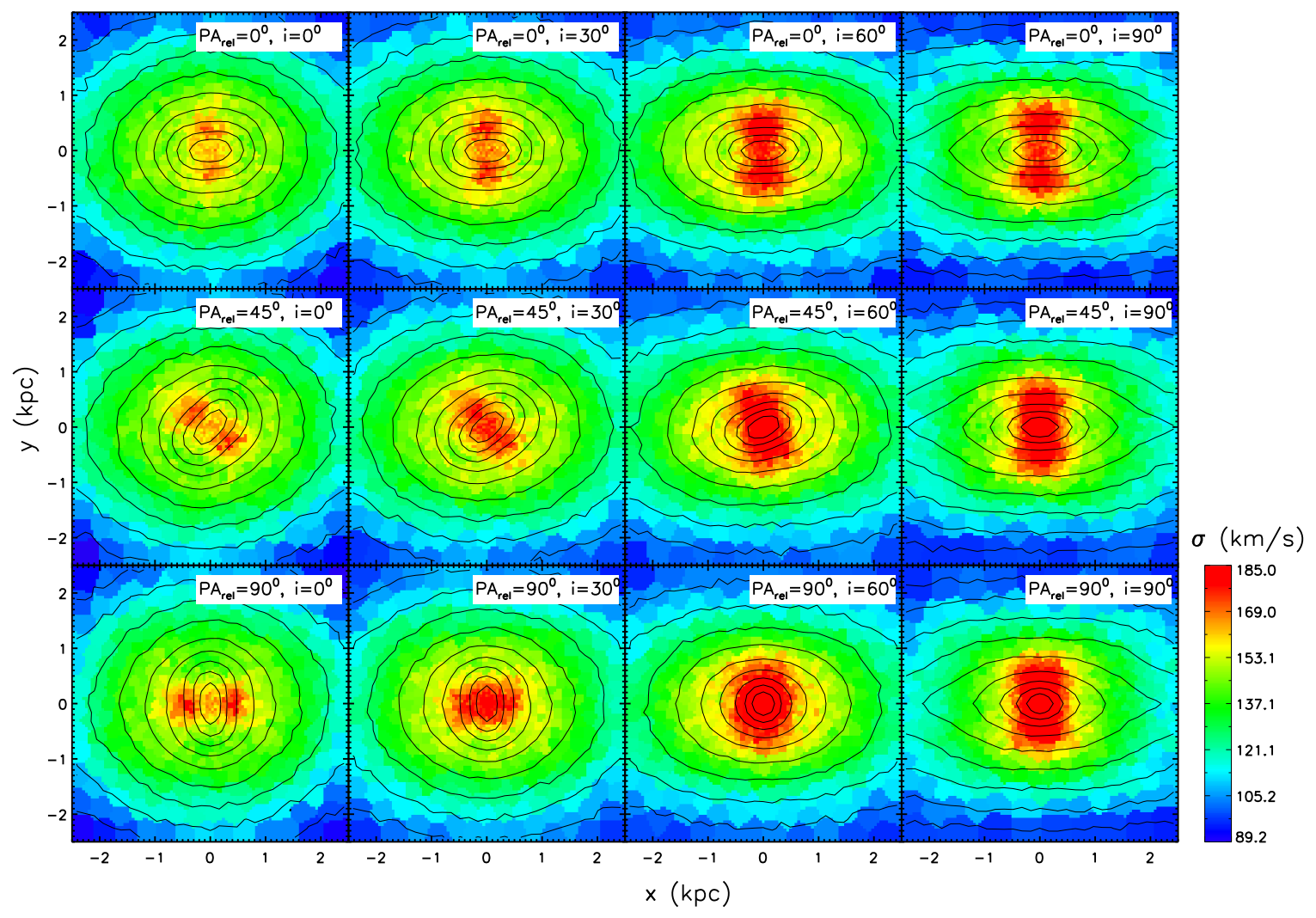

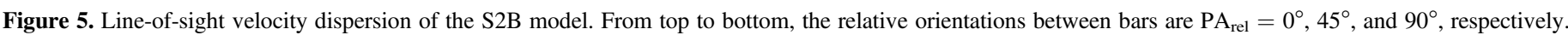

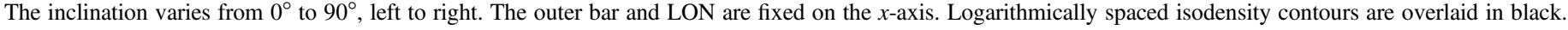

not find clear signs of the existence of decoupled inner bars in the form of central velocity twists. Moreover, we also did not find significant kinematic misalignments at the central regions of the SB at most inclinations and bar orientations, which is consistent with the IFU observations of early-type barred galaxies (Cappellari et al. 2007; Krajnović et al. 2011). The IFU observations show that the kinematic misalignment is always quite small $\left(\sim 5^{\circ}\right.$ level). We propose that such a result is due to the bars in early-type galaxies having large random motions.

Although the velocity twists are insignificant in the central regions, we notice that the observed S2Bs present consistent velocity twists at intermediate radii between the two bars. As shown in Figure 4, for the S2B model, some isovelocity contours (highlighted with white curves) are significantly distorted toward the central regions at intermediate radii $(R \sim 1.5 \mathrm{kpc})$ of the transition zone between the two bars. Independent of the relative orientation of the two bars and the inclination, such twists occur far from the photometric ends (the isodensity contour at level 0.8 ) of the inner bar. The transition zone where $\bar{v}$ twists occur is the region where the two bars are mixed and interacting. For NGC 2859, and NGC 2950, significant twists also appear at positions quite far from the end of the inner bar (highlighted with white curves), which is consistent with the S2B model. It is also worth noticing that the twists can be significantly asymmetric with respect to the LON. For example, the asymmetric twists in NGC 2950 can be clearly seen along the white isovelocity contours. On the upper side of the LON, the top-left arm is slightly distorted, while, on the lower side, the bottom-left arm has a nearly $90^{\circ}$ twist. The S2B model gives twists very consistent with those in NGC
2950. NGC 3941, which hosts only a weak inner bar, does not exhibit twists as significant as the model in their $\bar{v}$ fields, and the weak twists occur at positions close to the end of the inner bar. Without a nuclear disk in the model, such asymmetric twists may be caused by the non-axisymmetric motions in the transition zone of the S2B structure.

\section{2. $\sigma$-Humps}

\subsubsection{Basic Properties}

The $\sigma$ maps of the S2B model are shown in Figure 5 . Because varying the orientation of the outer bar does not lead to a significant difference, we fix the outer bar on the LON (the $x$-axis). The relative position angles of the two bars $\mathrm{PA}_{\text {rel }}$ are $\sim 0^{\circ}, 45^{\circ}$, and $90^{\circ}$, respectively, from top to bottom. From left to right, the disk is inclined from $i=0^{\circ}$ to $i=90^{\circ}$. In most cases, significant $\sigma$-humps appear along the minor axis of the inner bar. In contrast, in the SB model the elliptical $\sigma$ peak is aligned with the bar (the top row in Figure 6), which is consistent with previous SB models (Miller \& Smith 1979; Vauterin \& Dejonghe 1997), except for edge-on views.

As shown in Figure 5, $\sigma$-humps are closely associated with the inner bar. The basic properties of $\sigma$-humps are elaborated below. (1) $\sigma$-humps have a similar size as the inner bar. (2) The $\sigma$-humps are always present on the minor axis of the inner bar. In long-slit measurements, such $\sigma$-humps should appear as double-peaked or flat-topped distributions along the minor axis, while along the major axis $\sigma$ is relatively lower, forming $\sigma$ hollows. The difference can be a few tens of $\mathrm{km} \mathrm{s}^{-1}$ in this S2B model. (3) Projected properties of $\sigma$-humps are significantly affected by the orientation of the inner bar and the inclination 


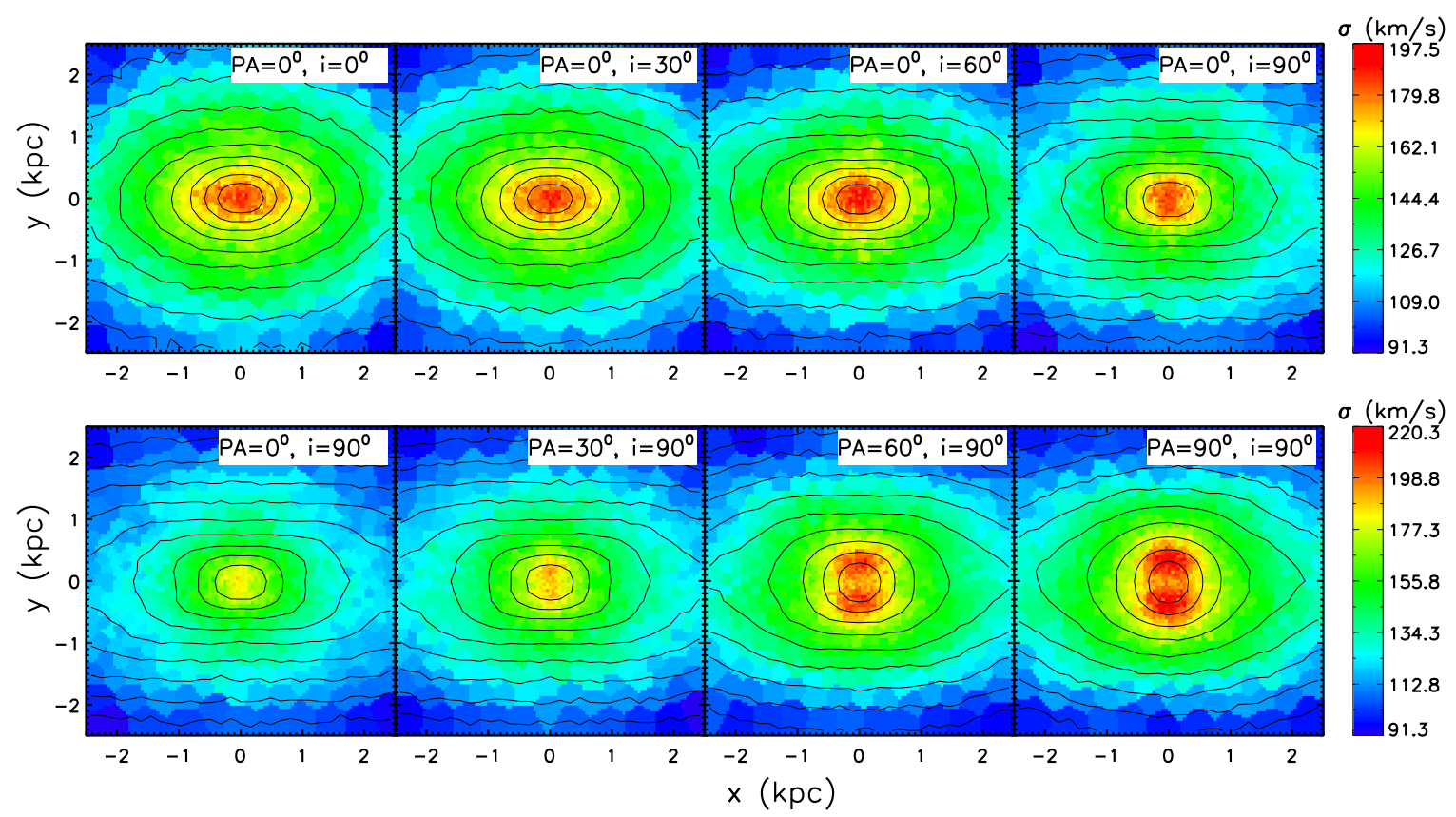

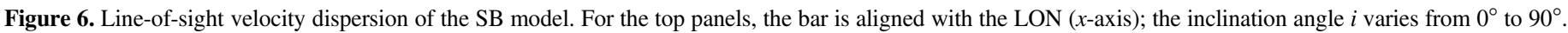
The bottom panels show the edge-on view of the SB model when the position angle of the bar varies from $0^{\circ}$ to $90^{\circ}$ with respect to the LON.

of the disk. In most cases, $\sigma$-humps are clearly visible. However, when the inner bar is nearly perpendicular to the LON, $\sigma$-humps are barely visible at intermediate inclinations $\left(i \sim 40^{\circ}-70^{\circ}\right)$. Then the difference in $\sigma$ between the minor and major axes becomes small, giving a nearly axisymmetric $\sigma$ distribution. (4) Around $\sigma$-humps/hollows, rings of enhanced $\sigma$ (by $\sim 10 \mathrm{~km} \mathrm{~s}^{-1}$ ) are present at most inclinations and orientations, except for edge-on views. (5) The amplitude of $\sigma$-humps oscillates in a similar way as the inner bar, i.e., $\sigma$-humps are weaker when the two bars are parallel $\left(\mathrm{PA}_{\text {rel }}=0^{\circ}\right)$ and stronger when the bars are perpendicular $\left(\mathrm{PA}_{\mathrm{rel}}=90^{\circ}\right)$. The physical origin of $\sigma$-humps is still unclear. de Lorenzo-Cáceres et al. (2008) proposed that $\sigma$-hollows might originate from a dynamically cold inner bar that is embedded in a relatively hotter (classical) bulge. Then the $\sigma$-humps/hollows are generated by the contrast between the hotter bulge and the cold bar. The S2B model presented here does not contain any classical bulge, but a $\mathrm{B} / \mathrm{P}$ bulge, forming from the internal buckling instability of the outer bar, which is also hotter than the inner bar. Further studies are needed to clarify whether $\sigma$ humps can be explained in terms of the contribution of bulges, as suggested by de Lorenzo-Cáceres et al. (2008).

Regardless of the relative orientation of the two bars, significant vertically extended $\sigma$ features (termed "vertical $\sigma$ humps") are present in edge-on views of both the S2B model (the rightmost panels of Figure 5) and the SB model (the bottom panels of Figure 6). For the S2B model, the vertical $\sigma$ humps in edge-on views are more extended and have larger values than $\sigma$-humps in face-on views. In the side-on view of the inner bar $\left(\mathrm{PA}_{\mathrm{rel}}=0^{\circ}, i=90^{\circ}\right), \sigma$ becomes lower close to the mid-plane than in the end-on view $\left(\mathrm{PA}_{\mathrm{rel}}=90^{\circ}, i=90^{\circ}\right)$. For the SB model, with increasing position angle of the bar from $0^{\circ}$ (side-on) to $90^{\circ}$ (end-on), the vertical $\sigma$-humps become more pronounced in the projected bar regions. Iannuzzi \& Athanassoula (2015) found similar features in edge-on views of their B/P bulge models hosting a single bar. Qin et al. (2015) also found a similar vertically extended $\sigma$ feature in their Milky
Way bar model (Shen et al. 2010). Falcón-Barroso et al. (2006) found that three of their five nearly edge-on galaxies (NGC 3623, NGC 4235, and NGC 5689) show such vertical $\sigma$ humps. In the ATLAS ${ }^{3 D}$ survey (Cappellari et al. 2011), we also find some edge-on galaxies exhibiting significant vertical $\sigma$-humps (NGC 2549, 3301, 3610, 4026, 4111, 4251, 4342, $4417,5308,5322$, and 5422). It is clear that both observations and simulations suggest that the vertical $\sigma$-humps are very common in edge-on galaxies. As suggested by Iannuzzi \& Athanassoula (2015), the peak value and extension of vertical $\sigma$ enhancements may be monotonic with the strength of the $\mathrm{B} / \mathrm{P}$ bulge. However, as shown in Figures 5 and 6 , both our S2B and SB models indicate that the existence of a bar can also significantly affect the properties of such vertically extended $\sigma$ features. However, because of the projection, it is hard to study the relation between bars and vertical $\sigma$-humps in the real edgeon galaxies. The physical origin and the relation with bars of such vertical $\sigma$ humps are still not clear; the vertical $\sigma$ humps do not seem to have the same origin as the $\sigma$-humps appearing in face-on views.

As shown in Figures 1 and 2, three S2Bs and one S2B candidate in the ATLAS ${ }^{3 D}$ and SAURON surveys show $\sigma$ humps, and are well matched by the model. NGC 2859 and NGC 3941 were included in the sample of de Lorenzo-Cáceres et al. (2008). For NGC 2859, the model also exhibits a similar moderate $\sigma$-ring feature around the $\sigma$-humps, as in the observation, which causes significant $\sigma$-hollows appearing at the ends of the inner bar. The $\sigma$-humps in NGC 3941 are not as significant as in the model, probably because the inner bar in NGC 3941 is much weaker than in the model, with rounder isodensity contours and a smoother $\bar{v}$ field. NGC 2950 and NGC 3384 are new examples of $\sigma$-hollow/hump galaxies. The orientation of the $\sigma$-humps in NGC 2950 is not accurately consistent with the model, which might be caused by the differences in their kinematic details between NGC 2950 and the numerical model. It is worth noticing that, around the $\sigma$ humps in NGC 2950 and the model, there are diffuse $\sigma$ spiral- 

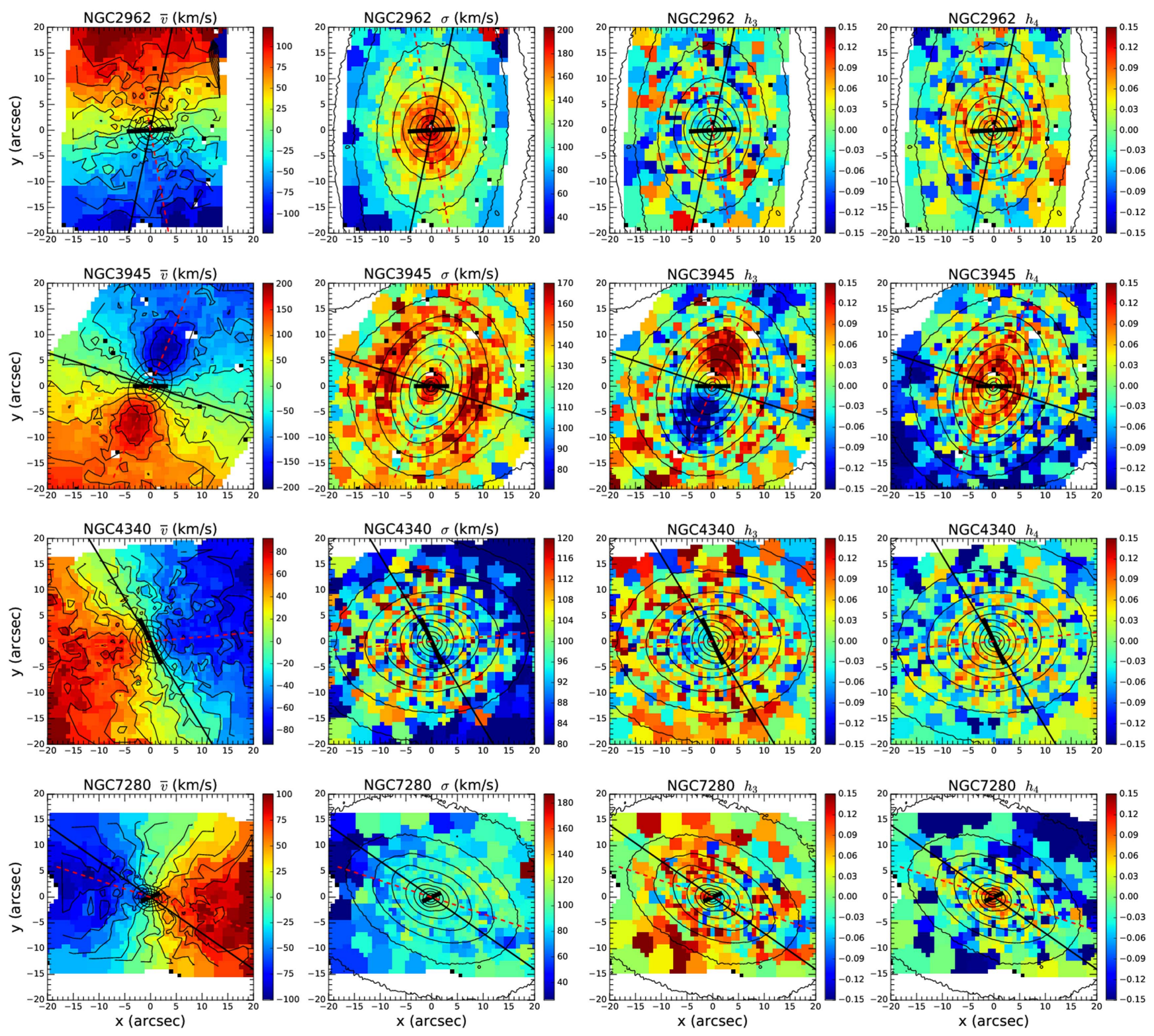

Figure 7. Kinematic maps for the remaining four S2B galaxies (NGC 2962, 3945, 4340, and 7280) in the ATLA. ${ }^{3 D}$ survey that do not show significant $\sigma$-humps.

like features, which might be similar with the $\sigma$ ring in NGC 2859. Using the Voronoi binning method with high enough $\mathrm{S} /$ $\mathrm{N}$, such $\sigma$ ring/spiral-like features are statistically significant kinematic features, which are still poorly understood. In conclusion, based on the simulation, we argue that $\sigma$-hollows are the same feature as $\sigma$-humps accompanied by $\sigma$ ring/spirallike features sometimes, viewed differently.

Finally, we briefly discuss the reason why the other four galaxies show no significant $\sigma$-humps. As shown in Figure 7, NGC 2962 and NGC 4340 are intermediately inclined, and their inner bars are almost perpendicular to the LON, $83^{\circ}$ and $70^{\circ}$, respectively. The model shows that the $\sigma$ humps are less noticeable in such conditions. For NGC 3945, as mentioned above, the inner regions are dominated by a large nuclear disk, which significantly affects the kinematic properties (Erwin et al. 2003; Cole et al. 2014), leaving no clear kinematic signatures of the inner bar. Finally, the inner bar in NGC 7280 seems too short to generate distinguishable kinematics. The S2B model may not match these galaxies very well.

\subsection{2. $\sigma$-humps in Aligned Double-barred Galaxies}

We have explored the kinematics of the simulations reported in $\mathrm{Du}$ et al. (2015). We find that $\sigma$-humps are not unique features of S2Bs. They are also present in galaxies hosting a single small-scale bar (i.e., nuclear-barred galaxies) and in aligned S2Bs, where the two bars have coupled into alignment leaving only a single bar. In Du et al. (2015), we showed that the coupling process distorts the isodensity contours to a peanut shape, which may be used to distinguish aligned S2B galaxies from normal single-barred galaxies. We plot the evolution of $\sigma$ humps during the coupling process in Figure 8. This aligned S2B model has the same initial conditions as the model in Figure 11 in Du et al. (2015), but its stellar mass $\left(M_{d}=1.0\right)$ is slightly lower. Before coupling, as shown in the top panels, the 


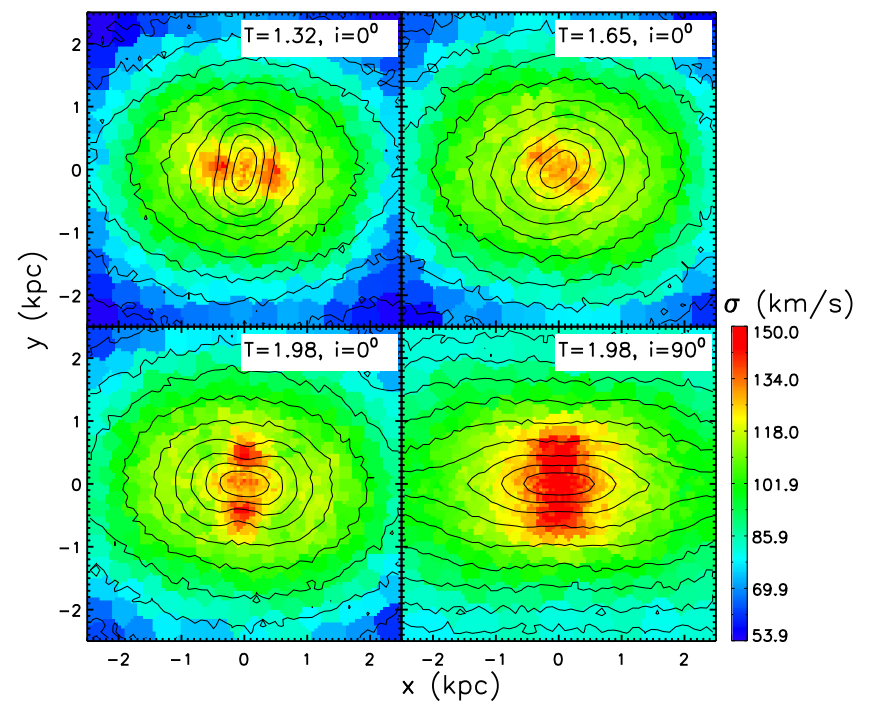

Figure 8. Co-evolution between $\sigma$-humps and the inner bar in an aligned S2B. The coupling process happens during $T=1.65-1.98$ Gyr. The peanut-shaped relic of the inner bar and $\sigma$-humps exist until the end of this simulation at $T \simeq 4.0 \mathrm{Gyr}$.

amplitude of the $\sigma$-humps gradually decreases with the strength of the inner bar. After the inner bar is trapped $(T \geqslant 1.98 \mathrm{Gyr}$, the bottom panels) by its outer counterpart, the peanut-shaped relic of the inner bar exhibits significant $\sigma$-humps on its minor axis. The co-evolution with the inner bar indicates that $\sigma$ humps may be used as a diagnostic of aligned S2Bs. We also expect that the amplitude of $\sigma$-humps will be affected by the bar strength in different models.

\subsection{Higher-order Moments: $h_{3}$ and $h_{4}$}

The higher-order Gauss-Hermite moments $h_{3}$ and $h_{4}$ describe the asymmetric and symmetric deviations, respectively, from a pure Gaussian (Gerhard 1993; van der Marel \& Franx 1993; Bender et al. 1994). It is well known that the LOSVD of an axisymmetric disk generally has a lower velocity tail due to the projected outer disk, yielding an anti-correlation of $h_{3}$ and $\bar{v}$. For barred galaxies, Bureau \& Athanassoula (2005) showed that in the bar regions, $h_{3}$ becomes correlated with $\bar{v}$ in edge-on views. They presented that the $h_{3}-\bar{v}$ correlation is an indication of the high-velocity tail created by the elongated orbits supporting the bar. The fourth-order Gauss-Hermite coefficient $h_{4}$ is negative when a distribution is broader than Gaussian and positive when it is more peaked. Debattista et al. (2005) showed that a B/P-shaped bulge generates a flat-topped LOSVD in face-on views.

In Figure 9, we present $h_{3}$ maps for the S2B model that cover the whole region of the outer bar $(\sim 7.5 \mathrm{kpc})$. The disk is inclined to $i=30^{\circ}, 60^{\circ}, 80^{\circ}$, and $90^{\circ}$ with respect to the $x$-axis. $\bar{v}$ is negative at $x<0$ and positive at $x>0$. It is not surprising that widespread $h_{3}-\bar{v}$ anti-correlation appears around the outer bar where LOSVDs are dominated by circular motions of the disk. With increasing $i$, the correlation between $h_{3}$ and $\bar{v}$ expands to all over the projected regions of the outer bar. This result is consistent with the SB cases studied in Bureau \& Athanassoula (2005). Closer to the center, the inner bar presents its own $h_{3}$ features. When the inner bar is nearly perpendicular to the LON (e.g., $T=3.50,3.54 \mathrm{Gyr}$ ), $h_{3}-\bar{v}$ changes to anti-correlation again at high inclinations $40^{\circ} \leqslant i \leqslant 90^{\circ}$, while when the inner bar is parallel to the LON (e.g., $T=3.47$ Gyr) there is no significant $h_{3}-\bar{v}$ anticorrelation. We propose that the $h_{3}-\bar{v}$ anti-correlation, appearing in the projected inner bar regions, is caused by the significant streaming motions in the inner bar which compose the high-velocity peak of the LOSVDs, thus generating $h_{3}-\bar{v}$ anti-correlation. In order to clarify the $h_{3}$ features clearly, we considered the non-Gaussian LOSVDs as bimodal profiles, which are assumed to be composed of two independent Gaussian components, i.e., the high $|\bar{v}|$ component and the low $|\bar{v}|$ component. Used to quantify the asymmetric deviation, $h_{3}$ is equal to zero in the cases in which the two components have exactly the same distribution. In the cases in which the low $|\bar{v}|$ component is stronger than the high $|\bar{v}|$ component, the LOSVD is composed of a main peak dominated by the low $|\bar{v}|$ component and a high $|\bar{v}|$ tail, in which case $h_{3}$ is correlated with $\bar{v}$. In contrast, if the high $|\bar{v}|$ component is stronger, the LOSVD is composed of a high $|\bar{v}|$ peak and a low $|\bar{v}|$ tail, yielding an anti-correlation of $h_{3}$ and $\bar{v}$. In the outer bar regions, the high-speed streaming motions in the outer bar generate the high $|\bar{v}|$ tail in the LOSVDs, which peak at low $|\bar{v}|$, thus generating $h_{3}-\bar{v}$ correlation. In the inner bar regions, because a large fraction of stars participate in the high-speed streaming motions in the inner bar, the high $|\bar{v}|$ component dominates the LOSVDs, thus $h_{3}$ can be anti-correlated with $\bar{v}$. Especially when the inner bar is perpendicular to the LON, the elongated motions in the inner bar well overlap with the line of sight to some extent, leading to the most prominent $h_{3}-\bar{v}$ anticorrelations. Because at $i=90^{\circ}$ the bars and the disk are superposed, the nuclear $h_{3}-\bar{v}$ anti-correlation is weakened.

As shown in Figure 10, the S2B also has noticeable $h_{4}$ features. In nearly face-on views, the most impressive $h_{4}$ feature is positive rings around the outer and the inner bars. Such positive $h_{4}$ rings around the inner bar were also noted by Shen \& Debattista (2009). With increasing inclination, positive $h_{4}$ rings become weaker and gradually disappear. Such features suggest that, compared with their surroundings, bars are more tightly bound at the mid-plane, thus the vertical velocity distribution is more peaked. In the intermediate regions, the negative $h_{4}$ probably corresponds to the $\mathrm{B} / \mathrm{P}$-shaped bulge.

We can also see complex non-Guassian features around the inner bars in observed S2Bs (Figures 1 and 2), i.e., $h_{3}-\bar{v}$ anticorrelations and positive $h_{4}$ rings. $h_{3}-\bar{v}$ anti-correlations have also been considered as tracers of nuclear disks (Bureau \& Athanassoula 2005). In all these S2Bs, anti-correlated $h_{3}$ are roughly aligned with the LON, while $h_{3}$ features have significant misalignments in the model. As mentioned above, both NGC 2950 and NGC 2859 probably host a nuclear disk that generates $h_{3}$ features well aligned with the LON. Therefore, though we cannot decompose nuclear disks and inner bars, the inner bar does provide an alternative explanation of nuclear $h_{3}-\bar{v}$ anti-correlations. The inner bars show positive $h_{4}$ rings in NGC 2859 and NGC 2950, which are consistent with the model, though the absolute value is much larger in the observations. NGC 3384 also shows some positive $h_{4}$ features and $h_{3}-\bar{v}$ anti-correlation at the bar regions, which are roughly consistent with the S2B model. For NGC 3941, positive $h_{4}$ is widely distributed all over the disk. At the central regions where the resolution is highest, we cannot identify an $h_{4}$ ring that is closely related to the inner bar, possibly because of the penalization on high-order Gauss-Hermite moments with the pPXF method, as mentioned in Section 3. 


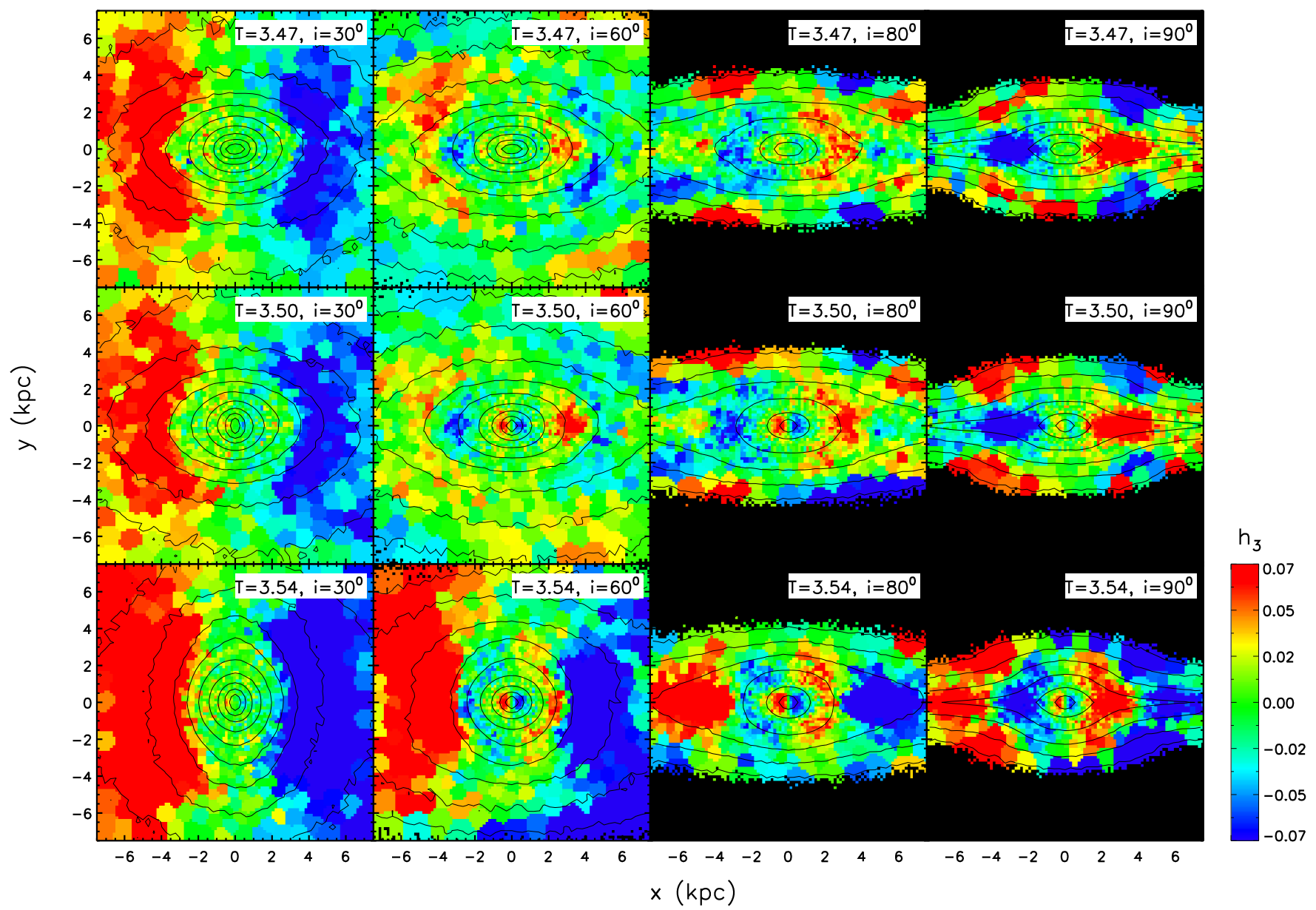

Figure 9. $h_{3}$ maps varying the orientation and inclination of the bars. The LON is fixed on the $x$-axis. From left to right: $i=30^{\circ}, 60^{\circ}, 80^{\circ}$, and $90^{\circ} . \bar{v}$ is negative at $x<0$ and positive at $x>0$. Isodensity contours are overlaid in black to show the orientation of two bars. At $T=3.50$ and 3.54 Gyr, the inner bar is perpendicular to the LON, while it is parallel to the LON at $T=3.47 \mathrm{Gyr}$. The outer bar is perpendicular to the LON at $T=3.54 \mathrm{Gyr}$, but parallel to the LON at $T=3.47$ and $3.50 \mathrm{Gyr}$. The color is set to black when the number of particles in one pixel $\left(\sim 150 \times 150 \mathrm{pc}^{2}\right)$ is less than 20 . Such regions cannot collect enough particles even with the Voronoi binning, and thus do not provide reliable kinematics.

\subsection{Intrinsic Kinematics}

As shown above, S2B galaxies have very different nuclear kinematics from single-barred galaxies. The inner bar in S2Bs exhibits many distinguishable properties that have never been found in a large single bar. Do these results suggest that the inner bar is essentially a different structure to normal bars? Because of the superposition of different components along the line of sight, the structural information is tangled in complex LOSVDs. To improve our understanding of S2Bs, we show the intrinsic kinematics of the outer (Figure 11) and inner (Figure 12) bars in the inertial frame, from left to right: $\overline{v_{R}}, \sigma_{R}, \overline{v_{\phi}}, \sigma_{\phi}$, and $\sigma_{z}$.

As we can see from Figure 11, the large-scale bars in the SB and S2B models have similar radial streaming motions, i.e., butterfly-shaped radial motions $\left(\overline{V_{R}}\right)$ extending to nearly half the length of the bars. As mentioned in Section 2.1, the rotation curve of the models here flattens at $v_{c} \sim 222 \mathrm{~km} \mathrm{~s}^{-1}$. It is clear that the stars in the outer disk rotate at $\sim v_{c}$, and the disk is thus dominated by nearly circular motions. The large-scale bars in both the SB and S2B models extend close to its corotation radius. At the ends of the large-scale bars, stars rotate at roughly $\sim v_{c}$. In the inner regions of the large-scale bars, the tangential velocity decreases significantly. Thus the random motions gradually become more important. We highlight the velocity dispersion contours at $0.5\left(\sigma_{\max }+\sigma_{\min }\right)$ with thick white curves. For large-scale bars, the radial velocity dispersion $\sigma_{R}$ is always aligned with the bar; the tangential velocity dispersion $\sigma_{\phi}$ is perpendicular to the bar. We compare the case when the two bars are parallel (the middle row) with when they are perpendicular (the bottom row). The relative orientation of the two bars has no significant effect on the intrinsic motions of the outer bar in the S2B model. Compared with the large-scale bar in the SB model (the top row), the outer bar in the S2B model has no distinguishable differences.

In Figure 12, we zoom into the central regions of Figure 11. As expected, the inner bar generates $\overline{v_{R}}$ and $\overline{v_{\phi}}$ features decoupled from the outer bar (the middle and bottom rows), while the bar in the SB model (the top row) acts as a solid body. For the inner bar in the S2B model, $\overline{v_{\phi}}$ shows significant local maxima along the minor axis. Such features clearly show that along the minor axis stars participate in the high-speed elongated motions, which is consistent with the expectation from the $h_{3}-\bar{v}$ anti-correlation discussed in Section 4.3. In spite of the differences in their sizes and pattern speeds, the inner bar in the S2B model and the large-scale bar in the SB model present similar $\sigma_{R}$ and $\sigma_{\phi}$ enhancements along the major and minor axis, respectively. From this point of view, inner bars have qualitatively similar intrinsic motions as large-scale bars, 


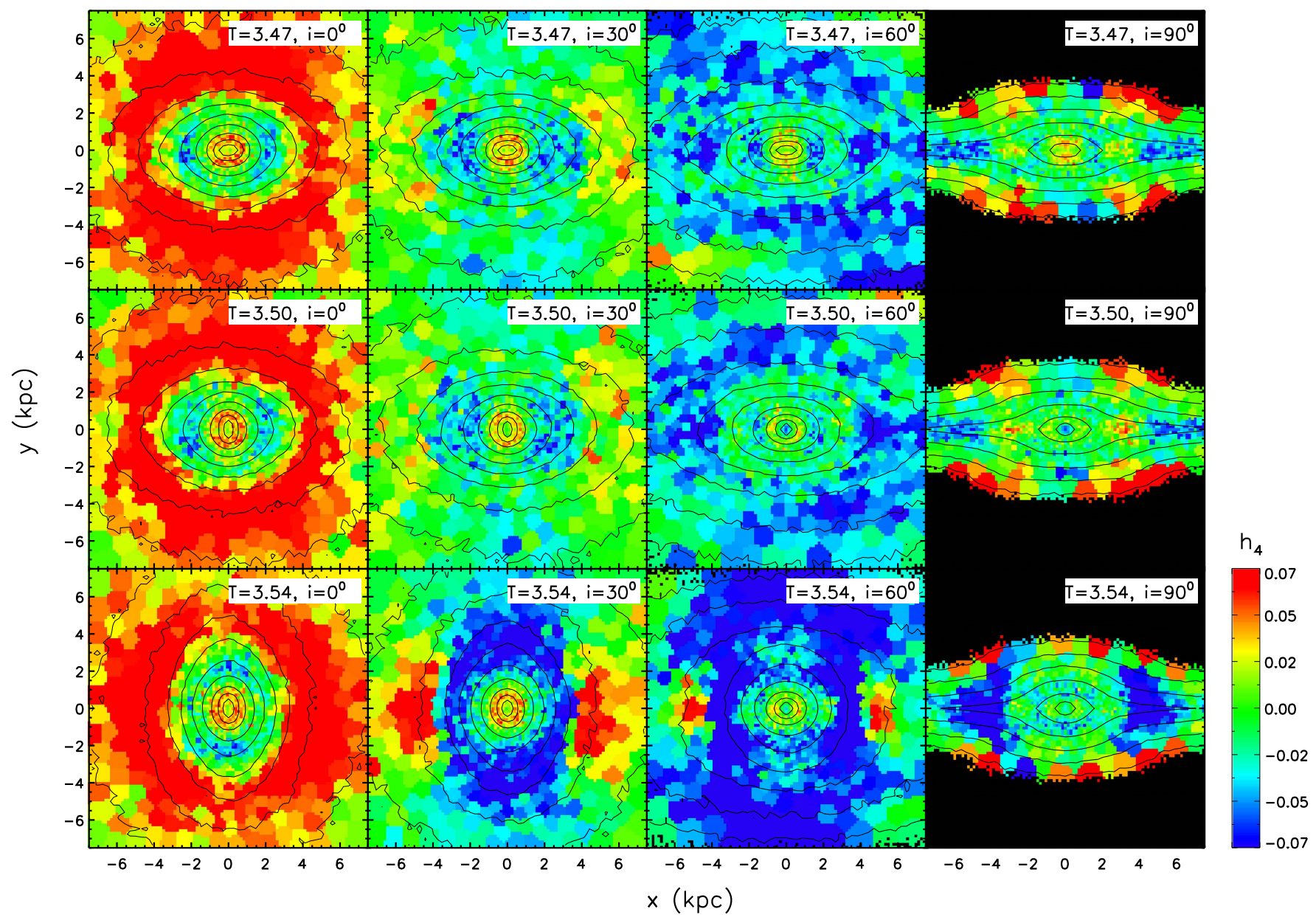

Figure 10. $h_{4}$ maps varying the orientations of the two bars and the inclination angle. The orientations of the LON and the two bars are the same as Figure 9 . From left to right, the disk is inclined at $i=30^{\circ}, 60^{\circ}, 80^{\circ}$, and $90^{\circ}$, respectively. Isodensity contours are overlaid in black.

being essentially scaled-down versions of normal large-scale bars. If that were the case, we would expect that the inner bar exhibits similar $\sigma$ features as large-scale bars and lacks $\sigma$-humps. However, as shown in the rightmost column in Figure 12, the inner bar presents $\sigma_{z}$-humps on its minor axis, while $\sigma_{z}$ smoothly decreases outward in the SB model. Therefore, the $\sigma$-humps seen in Figure 5 must be related to the $\sigma_{z}$-humps, as they are the only difference with the SB model.

To better understand $\sigma$-humps appearing in S2Bs, because the bar is a symmetric structure, it is more convenient to analyze the contributions of the parallel $\left(\sigma_{\|}\right)$and perpendicular $\left(\sigma_{\perp}\right)$ components with respect to the inner bar. In Figure 13 the inner bar is aligned with the $x$-axis; thus $\sigma_{\|}$and $\sigma_{\perp}$ are equal to $\sigma_{x}$ and $\sigma_{y}$, respectively. As shown here, $\sigma_{\perp}$ humps are present on the minor axis, while the elliptical $\sigma_{\|}$peak is aligned with the bar. When the inner bar is parallel to the LON, the line-ofsight velocity dispersion $\sigma_{\mathrm{LOS}}$ is mainly contributed by $\sigma_{\perp}$ and $\sigma_{z}$, by $\sigma_{\mathrm{LOS}}^{2}=\sigma_{\perp}^{2} \sin ^{2} i+\sigma_{z}^{2} \cos ^{2} i$. At small inclination, $\sigma_{\mathrm{LOS}}$ is determined by $\sigma_{z}$, while $\sigma_{\perp}$ becomes more and more important with increasing inclination. Because both $\sigma_{\perp}$ and $\sigma_{z}$ have significant humps at the minor axis, $\sigma$-humps are present at any inclination when the inner bar is parallel with the LON. When the inner bar is perpendicular to the LON, the $\sigma_{\mathrm{LOS}}$ is contributed by $\sigma_{\|}$and $\sigma_{z}$, by $\sigma_{\text {LOS }}^{2}=\sigma_{\|}^{2} \sin ^{2} i+\sigma_{z}^{2} \cos ^{2} i$. Because the $\sigma_{\|}$enhancement is aligned with the major axis of the inner bar, while $\sigma_{z}$ humps appear on the minor axis, the combination of $\sigma_{\|}$and $\sigma_{z}$ makes $\sigma_{\mathrm{LOS}}$ quite axisymmetric at intermediate inclinations. Thus $\sigma$-humps become barely visible for the case of $\mathrm{PA}_{\text {rel }}=90^{\circ}, i=60^{\circ}$ shown in Figure 5 .

Therefore, the $\sigma_{\perp}$ humps and $\sigma_{\|}$peak appearing on the minor and major axis, respectively, are normal kinematics of stellar bars. The properties of observed $\sigma_{\mathrm{LOS}}$-humps can be explained by the superimposition between $\sigma_{z}$-humps and such normal kinematics of bars. $\sigma_{z}$-humps play an important role in generating observable $\sigma$-humps. The physical origin of such $\sigma$ features will be studied in a follow-up study (Du et al. 2016, in preparation).

\section{THE KINEMATIC EFFECT OF A NUCLEAR DISK}

As suggested by recent numerical simulations (Du et al. 2015; Wozniak 2015), the inner bar may form from the bar instability of a dynamically cold nuclear disk, which forms from gas accumulation, followed by a starburst. This formation scenario indicates that a nuclear-disk-bar system where an inner bar is embedded in a rotation-dominated nuclear disk may be very common. In this paper, we show that the existence of an inner bar alone can explain the observed kinematics of S2Bs, especially $\sigma$-humps/hollows and positive $h_{4}$ rings. Our S2B model did not include the kinematic effect of a nuclear disk, which is well known as a mechanism for reducing $\sigma$ (e.g., Emsellem et al. 2001; Cole et al. 2014). There is no doubt that a 

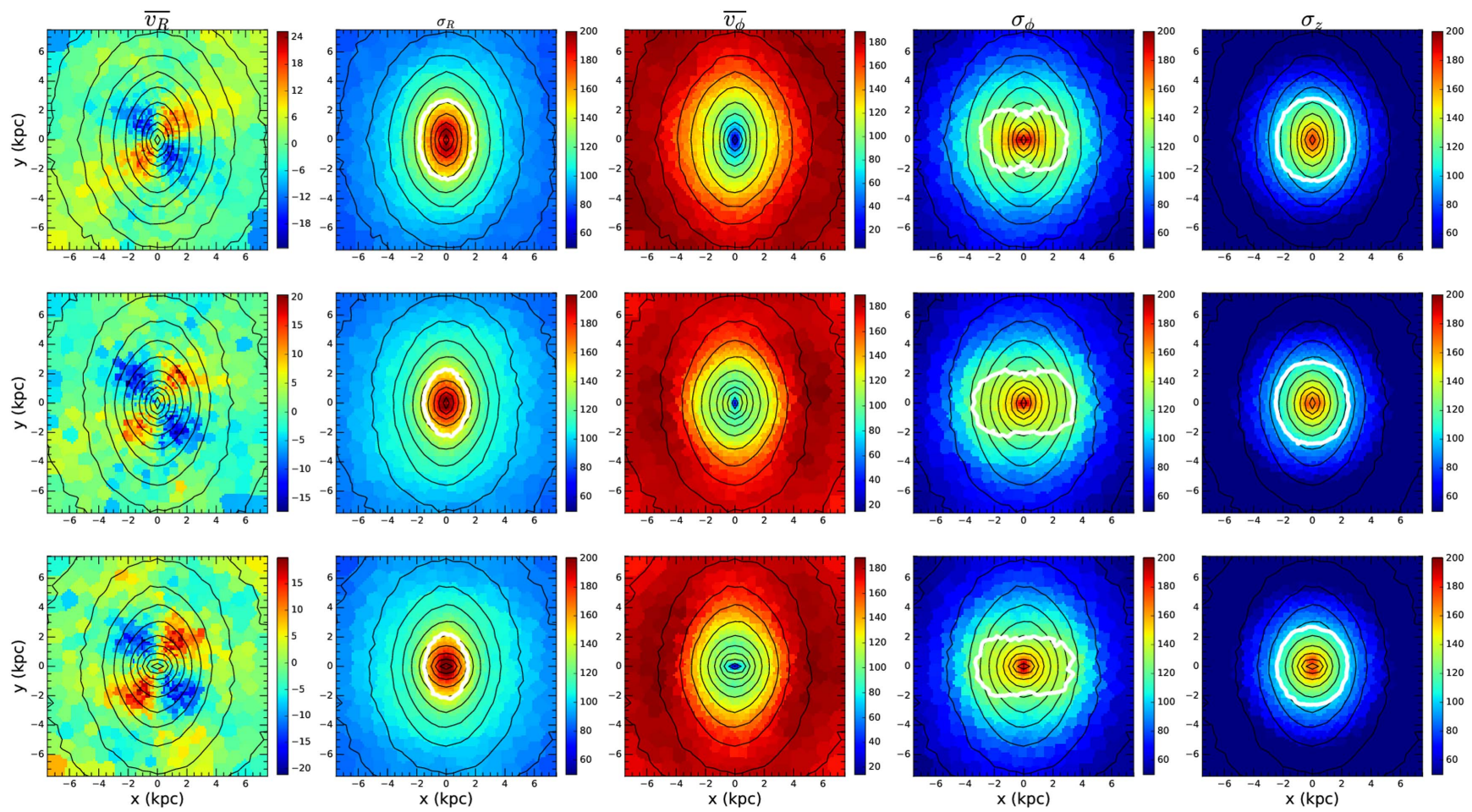

Figure 11. Face-on view of the kinematics in cylindrical coordinates, from left to right, $\overline{v_{R}}, \sigma_{R}, \overline{v_{\phi}}, \sigma_{\phi}$, and $\sigma_{z}$. The first row shows the SB model. The second and third rows show the parallel and perpendicular case, respectively, of two bars in the S2B model. The whole large-scale bars of S2B and SB models are covered. Isodensity contours are overlaid in black. To highlight the distribution of velocity dispersions, we also overlay their $0.5\left(\sigma_{\max }+\sigma_{\min }\right)$ contour with a thick white line.
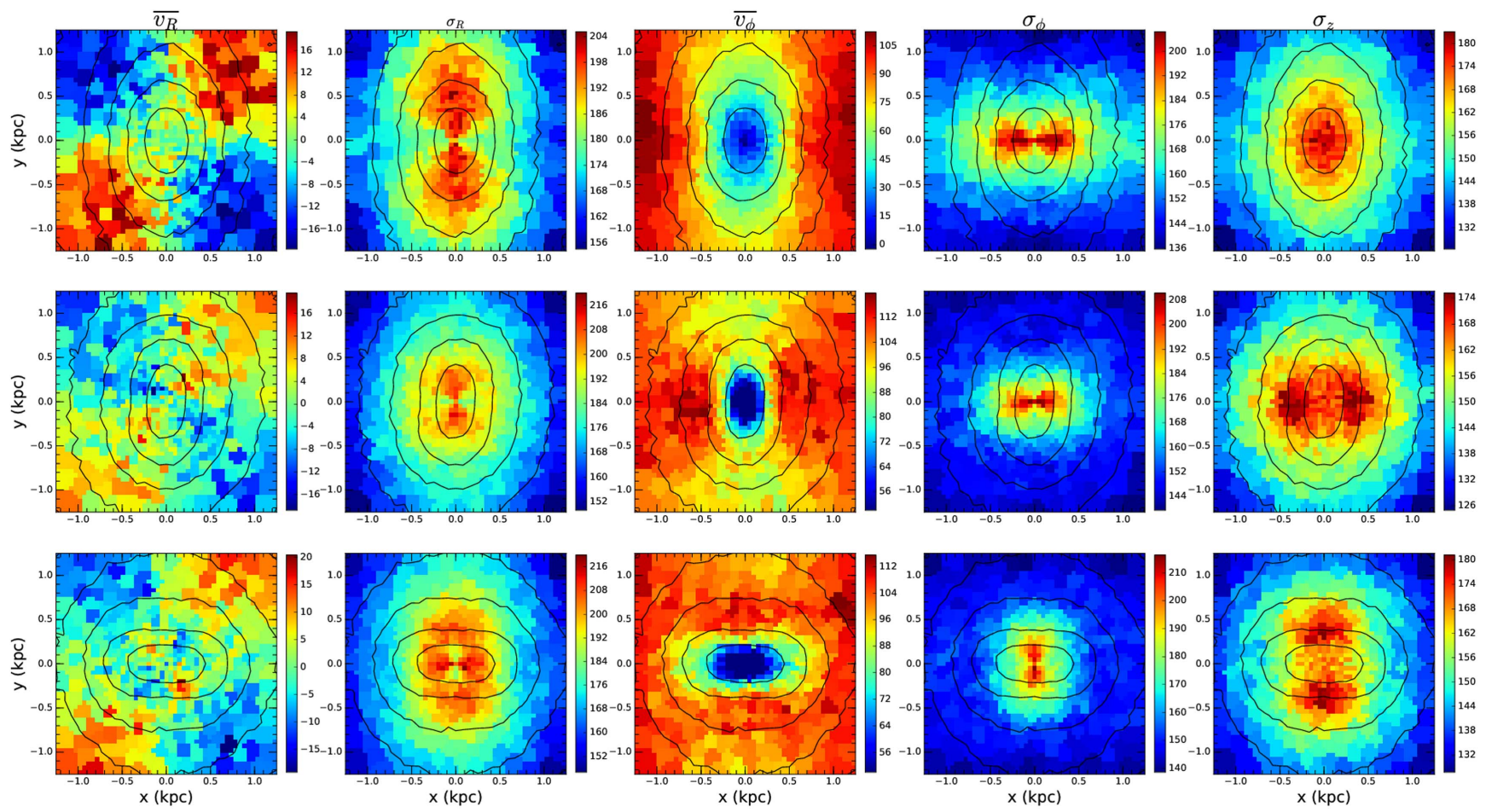

Figure 12. Same as Figure 11, zoomed into the central regions dominated by the inner bar.

dynamically cooler nuclear disk, forming from the inflow of gas, can cause central $\sigma$ drops. Being embedded in a dynamically hotter bar or bulge, $\sigma$ is lower over the projected regions of the nuclear disk, while beyond the nuclear disk $\sigma$ is relatively larger. As shown in Figure 7, NGC 3945 is a prototypical example of the kinematics of a strong nuclear disk. Cole et al. (2014) compared this galaxy extensively with their $\mathrm{N}$-body+gas simulation. Within the projected nuclear disk, the reduced $\sigma$ value in NGC 3945 is quite flat except for the very central regions, which is consistent with the $N$-body+gas 

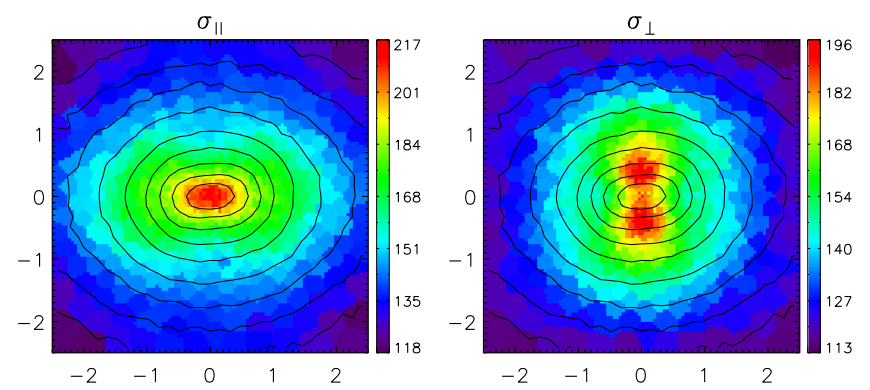

Figure 13. Face-on view of velocity dispersions in the parallel $\left(\sigma_{\|}\right)$and perpendicular $\left(\sigma_{\perp}\right)$ directions with respect to the inner bar in the S2B model. Both the outer and the inner bars are aligned with the $x$-axis.

simulation described in Cole et al. (2014). The relatively larger $\sigma$ on the minor axis may give an impression of " $\sigma$-humps" perpendicular to the LON.

Without a nuclear disk, the kinematics of the S2B model are mainly dominated by the velocity dispersion. For NGC 2859 and NGC 2950, the nuclear disk causes significant local minima and maxima in $\bar{v}$ fields and an $h_{3}-\bar{v}$ anti-correlation, while we cannot see any clear effects of the nuclear disk in the $\sigma$ maps. The $\sigma$ features can be well matched by the S2B model without a nuclear disk, which suggests that such nuclear disks may not efficiently reduce the $\sigma$ value in these S2Bs. The nuclear disk in NGC 3384, if it exists, seems even weaker than in NGC 2950, thus it may not be sufficient for forming $\sigma$ hollows (or $\sigma$-humps) as large as $30 \mathrm{~km} \mathrm{~s}^{-1}$. Although we cannot rule out the scenario that $\sigma$-humps are caused by a nuclear disk, the S2B model does share similar kinematic features with NGC 3384. It is reasonable to consider NGC 3384 as a potential S2B candidate. Further numerical simulations are required to better quantify the kinematic effect of a dynamically cold nuclear disk, especially for nuclear-diskbar systems.

\section{SUMMARY}

This study sheds new light on the kinematic properties of double-barred galaxies. Using well-resolved, self-consistent simulations, we have studied the kinematic properties of double-barred galaxies in comparison to single-barred galaxies. By quantifying the LOSVDs with Gauss-Hermite moments, we find that many significant kinematic features are closely associated with the inner bar. The most notable feature is $\sigma$ humps that appear on the minor axis of inner bars, matching well with the integral-field observations of the stellar kinematics from the ATLAS ${ }^{3 D}$ and SAURON surveys. Accompanied by $\sigma$-ring/spiral-like features, $\sigma$-humps may help to explain the ubiquitous $\sigma$-hollows in S2Bs seen in previous observations. Generally, $\sigma$-humps evolve and oscillate together with the inner bar. Based on the analysis of intrinsic motions of bars, we show that the inner bar is essentially a scale-down version of normal large-scale bars from the kinematic point of view. The only difference is the $\sigma_{z}$-humps appearing on the minor axis of the inner bar. Combined with $\sigma_{\|}$enhancements and $\sigma_{\perp}$ humps produced in normal bars, $\sigma_{z}$-humps are the key to generating the observed $\sigma$-humps in S2Bs.

The isovelocity contours are significantly distorted. However, at the central regions, the kinematic major axis is only slightly distorted toward the opposite direction with respect to the inner bars. The most significant asymmetric twists are present at intermediate radii, in the transition region between the two bars instead of the photometric end of the inner bar. Because of the elongated streaming motions in bars, some nonGaussian features appear. The outer bar exhibits an $h_{3}-\bar{v}$ correlation, as expected. However, in the central regions, $h_{3}$ becomes anti-correlated with $\bar{v}$ as a result of the increasing dominance of the inner bar. The inner bar exhibits significant positive $h_{4}$ rings in nearly face-on cases, suggesting that the inner bar has a sharply peaked $v_{z}$ distribution.

M.D. thanks the Jeremiah Horrocks Institute of the University of Central Lancashire for their hospitality during a three-month visit while this paper was in progress. Hospitality at APCTP during the 7th Korean Astrophysics Workshop is kindly acknowledged. We thank Peter Erwin for constructive discussions and providing us with the WIYN images of the target galaxies, and Adriana de Lorenzo-Cáceres and Martin Bureau for constructive comments and discussions on the manuscript. M.D. also warmly thanks Sarah Bird for help with the language of this paper. The research presented here is partially supported by the 973 Program of China under grant no. 2014CB845700, by the National Natural Science Foundation of China under grant nos.11333003 and 11322326, and by the Strategic Priority Research Program "The Emergence of Cosmological Structures" (no. XDB09000000) of the Chinese Academy of Sciences. We acknowledges support from a Newton Advanced Fellowship awarded by the Royal Society and the Newton Fund, and from the CAS/SAFEA International Partnership Program for Creative Research Teams. This work made use of the facilities of the Center for High Performance Computing at Shanghai Astronomical Observatory. V.P.D. is supported by STFC Consolidated grant \# ST/J001341/1. V.P. D. was also partially supported by the Chinese Academy of Sciences President's International Fellowship Initiative Grant (No. 2015VMB004). M.C. acknowledges support from a Royal Society University Research Fellowship.

\section{REFERENCES}

Bender, R., Saglia, R. P., \& Gerhard, O. E. 1994, MNRAS, 269, 785

Bureau, M., \& Athanassoula, E. 2005, ApJ, 626, 159

Buta, R., \& Crocker, D. A. 1993, AJ, 105, 1344

Cappellari, M., \& Copin, Y. 2003, MNRAS, 342, 345

Cappellari, M., \& Emsellem, E. 2004, PASP, 116, 138

Cappellari, M., Emsellem, E., Bacon, R., et al. 2007, MNRAS, 379, 418

Cappellari, M., Emsellem, E., Krajnović, D., et al. 2011, MNRAS, 413, 813

Chevalier, R. A., \& Furenlid, I. 1978, ApJ, 225, 67

Cole, D. R., Debattista, V. P., Erwin, P., Earp, S. W. F., \& Roškar, R. 2014, MNRAS, 445, 3352

Corsini, E. M., Debattista, V. P., \& Aguerri, J. A. L. 2003, ApJL, 599, L29

de Lorenzo-Cáceres, A., Falcón-Barroso, J., \& Vazdekis, A. 2013, MNRAS, 431, 2397

de Lorenzo-Cáceres, A., Falcón-Barroso, J., Vazdekis, A., \& Martínez-Valpuesta, I. 2008, ApJL, 684, L83

de Lorenzo-Cáceres, A., Vazdekis, A., Aguerri, J. A. L., Corsini, E. M., \& Debattista, V. P. 2012, MNRAS, 420, 1092

Debattista, V. P., Carollo, C. M., Mayer, L., \& Moore, B. 2005, ApJ, 628, 678 Debattista, V. P., \& Shen, J. 2007, ApJL, 654, L127

Du, M., Shen, J., \& Debattista, V. P. 2015, ApJ, 804, 139

Emsellem, E., Cappellari, M., Peletier, R. F., et al. 2004, MNRAS, 352, 721

Emsellem, E., Greusard, D., Combes, F., et al. 2001, A\&A, 368, 52

Englmaier, P., \& Shlosman, I. 2004, ApJL, 617, L115

Erwin, P. 2004, A\&A, 415, 941

Erwin, P. 2005, MNRAS, 364, 283

Erwin, P., Beltrán, J. C. V., Graham, A. W., \& Beckman, J. E. 2003, ApJ, 597, 929

Erwin, P., Saglia, R. P., Fabricius, M., et al. 2015, MNRAS, 446, 4039

Erwin, P., \& Sparke, L. S. 2002, AJ, 124, 65

Erwin, P., \& Sparke, L. S. 2003, ApJS, 146, 299 
Falcón-Barroso, J., Bacon, R., Bureau, M., et al. 2006, MNRAS, 369, 529

Fisher, D. B., \& Drory, N. 2010, ApJ, 716, 942

Friedli, D., \& Martinet, L. 1993, A\&A, 277, 27

Gerhard, O. E. 1993, MNRAS, 265, 213

Heller, C. H., Shlosman, I., \& Athanassoula, E. 2007, ApJL, 657, L65

Iannuzzi, F., \& Athanassoula, E. 2015, MNRAS, 450, 2514

Krajnović, D., Emsellem, E., Cappellari, M., et al. 2011, MNRAS, 414, 2923

Laine, S., Shlosman, I., Knapen, J. H., \& Peletier, R. F. 2002, ApJ, 567, 97

Maciejewski, W., \& Athanassoula, E. 2007, MNRAS, 380, 999

Maciejewski, W., \& Athanassoula, E. 2008, MNRAS, 389, 545

Maciejewski, W., \& Small, E. E. 2010, ApJ, 719, 622

Maciejewski, W., \& Sparke, L. S. 1997, ApJL, 484, L117

Maciejewski, W., \& Sparke, L. S. 2000, MNRAS, 313, 745

Méndez-Abreu, J., Debattista, V. P., Corsini, E. M., \& Aguerri, J. A. L. 2014, A\&A, 572, AA25

Miller, R. H., \& Smith, B. F. 1979, ApJ, 227, 785

Moiseev, A. V., \& Mustsevoi, V. V. 2000, AstL, 26, 565

Moiseev, A. V., Valdés, J. R., \& Chavushyan, V. H. 2004, A\&A, 421, 433
Petitpas, G. R., \& Wilson, C. D. 2004, ApJ, 603, 495

Qin, Y., Shen, J., Li, Z.-Y., et al. 2015, ApJ, 808, 75

Rautiainen, P., \& Salo, H. 1999, A\&A, 348, 737

Rautiainen, P., Salo, H., \& Laurikainen, E. 2002, MNRAS, 337, 1233

Romeo, A. B., \& Fathi, K. 2015, MNRAS, 451, 3107

Saha, K., \& Maciejewski, W. 2013, MNRAS, 433, L44

Sarzi, M., Falcón-Barroso, J., Davies, R. L., et al. 2006, MNRAS, 366, 1151

Sellwood, J. A. 2014, arXiv:1406.6606

Sellwood, J. A., \& Valluri, M. 1997, MNRAS, 287, 124

Shen, J., \& Debattista, V. P. 2009, ApJ, 690, 758

Shen, J., Rich, R. M., Kormendy, J., et al. 2010, ApJL, 720, L72

Shlosman, I., Begelman, M. C., \& Frank, J. 1990, Natur, 345, 679

Shlosman, I., Frank, J., \& Begelman, M. C. 1989, Natur, 338, 45

Shlosman, I., \& Heller, C. H. 2002, ApJ, 565, 921

van der Marel, R. P., \& Franx, M. 1993, ApJ, 407, 525

Vauterin, P., \& Dejonghe, H. 1997, MNRAS, 286, 812

Wozniak, H. 2015, A\&A, 575, A7 